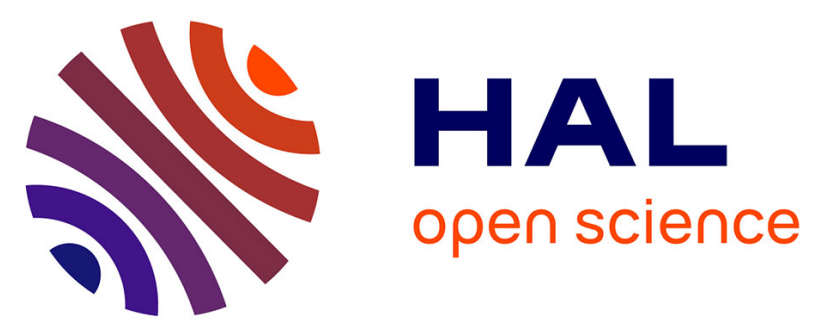

\title{
Numerical study of natural convection and acoustic waves using the lattice Boltzmann method
}

Jaouad Benhamou, Mohammed Jami, Ahmed Mezrhab, Valéry Botton, Daniel Henry

\section{- To cite this version:}

Jaouad Benhamou, Mohammed Jami, Ahmed Mezrhab, Valéry Botton, Daniel Henry. Numerical study of natural convection and acoustic waves using the lattice Boltzmann method. Heat Transfer Asian Research, 2020, 49 (6), pp.3779-3796. 10.1002/htj.21800 . hal-02923798

\section{HAL Id: hal-02923798 \\ https://hal.science/hal-02923798}

Submitted on 13 Nov 2020

HAL is a multi-disciplinary open access archive for the deposit and dissemination of scientific research documents, whether they are published or not. The documents may come from teaching and research institutions in France or abroad, or from public or private research centers.
L'archive ouverte pluridisciplinaire HAL, est destinée au dépôt et à la diffusion de documents scientifiques de niveau recherche, publiés ou non, émanant des établissements d'enseignement et de recherche français ou étrangers, des laboratoires publics ou privés. 


\title{
Numerical study of natural convection and acoustic waves using Lattice Boltzmann Method
}

\author{
J. Benhamou ${ }^{a}$, M. Jami ${ }^{a 1}$, A. Mezrhab ${ }^{a}$, V. Botton ${ }^{b}$, D. Henry ${ }^{b}$ \\ a. Laboratoire de Mécanique et Energétique, Faculté des Sciences, Université Mohammed \\ Premier, 60000 Oujda, Morocco \\ b. Laboratoire de Mécanique des Fluides et d'Acoustique, CNRS/Université de Lyon, Ecole \\ Centrale de Lyon/Université Lyon 1/INSA Lyon, ECL, 36 Avenue Guy de Collongue, 69134 \\ Ecully Cedex, France
}

\begin{abstract}
In this paper, the Lattice Boltzmann Method (LBM) is used to study the acoustic waves propagation inside a differentially heated square enclosure filled with air. The waves are generated by a point sound source located at the center of this cavity. The main aim of this simulation is to simulate the interaction between the thermal convection and the propagation of these acoustic waves. The results have been validated with those obtained in the literature and show that the effect of natural convection on the acoustic waves propagation is almost negligible for low Rayleigh numbers ( $\mathrm{Ra} \leq 10^{4}$ ), begins to appear when the Rayleigh number begins to become important $\left(\mathrm{Ra} \geq 10^{5}\right)$ and it becomes considerable for large Rayleigh numbers $\left(\mathrm{Ra} \geq 10^{6}\right)$ where the thermal convection is important.
\end{abstract}

Keywords: Acoustic wave, free convection, lattice Boltzmann method.

\section{Introduction}

Theoretically, LBM is an old method. However, numerically, this is a relatively new method. It is used at the mesoscopic scale to simulate different physical phenomena. It is derived from the kinetic theory and its origin returns to Lattice Gas Automata [1-3].

The LBM is one of the useful tools for simulating wave propagation in complex media [4]. Several researchers have used this method to study different waves, such as acoustic wave, elastic waves, aeroacoustic wave, shock wave, acoustic streaming and so on.

Beginning with shock wave, Yan et al. [5] proposed a lattice Boltzmann scheme to simulate the compressible Euler equations. They showed that the proposed model can be used to study numerically the shock wave and contact discontinuity. Xiao [6] proposed a new lattice Boltzmann approach to investigate the propagation of the shock wave in elastic solids.

For elastic waves, Frantziskonis [7] used the LBM formulation to study the viscoelastic fluids with volumetric and shear viscoelasticity. O'Brien et al. [8] outlined a new 2D and 3D lattice Boltzmann solutions to the elastic wave equation in a Poisson solid using a regular LBM lattice.

Regarding aeroacoustic wave, Li et al. [9] selected the circular acoustic pulse propagation, the onedimensional acoustic pulse propagation, the propagation of acoustic and entropy pulses in a uniform stream to test the one step lattice Boltzmann method aeroacoustics simulation. 
Marié et al. [10] studied and compared two kinds of schemes for computational aeroacoustics: the lattice Boltzmann method and the classical numerical approach based on high order schemes for Navier-Stokes-like equations.

For acoustic streaming, Haydock et al. [11] employed the lattice Boltzmann method to simulate the acoustic streaming created by the interaction between a sound wave and a boundary. They compared the numerical results found with the analytic results for Rayleigh streaming within the appropriate limits and showed how deviating from those limits can affect the streaming. They also modeled the acoustic flux induced by the attenuation produced by a progressive wave [12].

Finally, for acoustic wave, Buick et al. [13] used the lattice Boltzmann method based on the BGK scheme to study the sound waves propagation in systems where the variation of the density is small in relation to the mean density. Salomons et al. [14] employed the lattice Boltzmann tool to simulate the propagation of the sound on porous and non-porous soils, in free field, in a windy atmosphere and on an acoustic barrier. Recently, the lattice Boltzmann for simulating wave propagation in viscous media was used by Xia et al. [15].

The interaction of thermal phenomena with the sound waves propagation is another problem in acoustics. There are few studies on acoustic-thermal coupling problems. Among these works, we will mention that of Vincenti et al. [16], who studied the sound waves propagation in a semi-infinite expanse of radiant gases on one side of an infinite and radiating planar wall. They found a particular solution for the case of sinusoidal oscillations in both position and temperature of the wall. Park et al. [17] studied the propagation of the sound wave inside a radiating gas medium with the solid particles in suspension. Tarau et al. [18] numerically studied the propagation of acoustic waves inside regions of a gas where the temperature distribution is non-uniform. They determined the temperature gradients impact on the sound wave parameters. Nabavi et al. [19] experimentally investigated the effects of transverse gradient of temperature on streaming velocity and acoustic fields inside a rectangular enclosure which is filled with gas and subjected to acoustic standing wave. Another experimental study was carried out by Saint Ellier et al. [20]. It consists in showing the effect of an axial gradient of temperature in a cylindrical resonant cavity. Recently, Červenka et al. [21] numerically studied the acoustic streaming in a cylindrical resonator filled with air and with a gradient of temperature imposed on its walls. They showed that the streaming profile is strongly influenced by the convective heat transfer, even when the gradient of the temperature is weak.

The originality of the present work focuses on modelling the coupling of thermal convection with acoustic waves generated by a point sound source located at the center of a square cavity asymmetrically heated filled with air. The numerical approach uses a D2Q9-MRT scheme for fluid velocity variables and for the acoustic phenomenon, and a D2Q5-MRT scheme to determine the temperature field. Various physical parameters such as the fluid density, the amplitude of the acoustic source, and the Rayleigh number are studied numerically to evaluate their effects on the natural convection and the sound waves propagation. Thereby, this methodology constitutes a remarkable contribution to the understanding of the interaction between natural convection and acoustic waves. It could also be used in the future by researchers who wish to understand the behavior of wave propagation in heat- flow media. The paper is organized as follows: in the next section the description of the LBM is given, in the third section boundary conditions are reported, and then the numerical results and analyses concerning the parametrical study are presented in the Section 4. Finally conclusions of numerical study are done in Section 5. 


\section{Lattice Boltzmann method}

The lattice Boltzmann method based on Multi-Relaxation Time (MRT) is used to simulate the coupling between heat transfer and wave propagation. This MRT model is preferred because it presents high precision and stability compared to Single Relaxation Time (SRT) model [22].

D2Q9-MRT scheme is applied to determine the macroscopic quantities such as the fluid density, the velocities and the pressure. D2Q5-MRT scheme is used to solve the temperature field.

\subsection{D2Q9-MRT scheme for the acoustic phenomenon}

For the acoustic calculations, we have used the D2Q9 model. For this model, there are nine discrete velocities $c_{i}$ as shown in Fig.1. The temporal evolution of the fluid state is described by the following Boltzmann equation [23]:

$$
f_{i}\left(x_{i}+c_{i} \Delta t, t+\Delta t\right)-f_{i}\left(x_{i}, t\right)=\Omega_{i}
$$

where $f_{i}, c_{i}, \Delta t$ and $\Omega_{i}$ are the particle distribution, the lattice velocities, the time step and the collision operator, respectively.

$$
c_{i}=\left\{\begin{array}{cl}
(0,0) & i=0 \\
(1,0) \mathrm{c},(0,1) c,(-1,0) \mathrm{c},(0,-1) \mathrm{c} & i=1, \ldots, 4 \\
(1,1) \mathrm{c},(-1,1) c,(-1,-1) \mathrm{c},(1,-1) \mathrm{c} & i=5, \ldots, 8
\end{array}\right.
$$

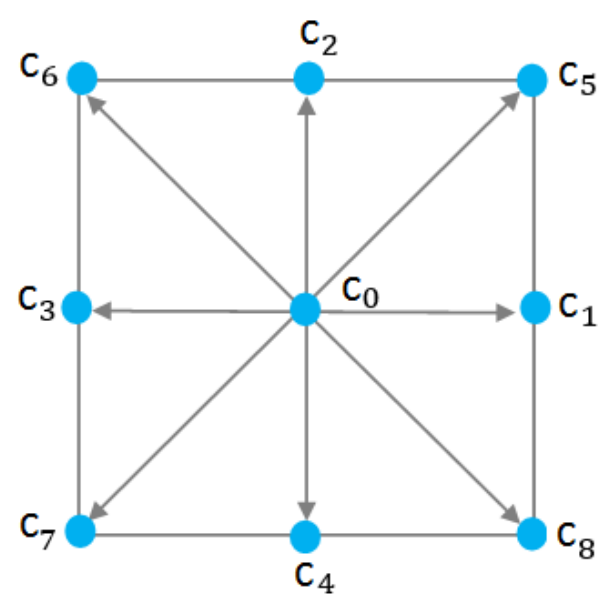

Fig. 1. D2Q9 lattice.

The LBM parameter $\mathrm{c}$ represents the streaming speed. It is defined as $\delta_{x} / \delta_{t}$ where $\delta_{t}$ and $\delta_{x}$ are respectively the time step and streaming length. They are taken equal to 1 .

For the MRT-LBM, the collision operator can be generalized as in [23,24]:

$$
\Omega_{i}=-M^{-1} S\left[m_{i}-m_{i}^{e q}\right]
$$

where $M^{-1}$ is the inverse matrix of the transformation matrix $M$ which maps the vector $f=$ $\left(f_{0}, f_{1}, f_{2}, \ldots, f_{8}\right)^{T}$ to the vector $m=\left(m_{0}, m_{1}, m_{2}, \ldots, m_{8}\right)^{T}$ by the following linear transformations:

$$
m=M f \quad \text { and } \quad f=M^{-1} m
$$




$$
M=\left(\begin{array}{rrrrrrrrr}
1 & 1 & 1 & 1 & 1 & 1 & 1 & 1 & 1 \\
-4 & -1 & -1 & -1 & -1 & 2 & 2 & 2 & 2 \\
4 & -2 & -2 & -2 & -2 & 1 & 1 & 1 & 1 \\
0 & 1 & 0 & -1 & 0 & 1 & -1 & -1 & 1 \\
0 & -2 & 0 & 2 & 0 & 1 & -1 & -1 & 1 \\
0 & 0 & 1 & 0 & -1 & 1 & 1 & -1 & -1 \\
0 & 0 & -2 & 0 & 2 & 1 & 1 & -1 & -1 \\
0 & 1 & -1 & 1 & -1 & 0 & 0 & 0 & 0 \\
0 & 0 & 0 & 0 & 0 & 1 & -1 & 1 & -1
\end{array}\right)
$$

$S$ is the diagonal relaxation matrix of fluid flow, it can be written as:

$$
\mathrm{S}=\operatorname{diag}\left(s_{0}, s_{1}, s_{2}, s_{3}, s_{4}, s_{5}, s_{6}, s_{7}, s_{8}\right)
$$

In this work, we choose $s_{0}=s_{3}=s_{5}=1, s_{1}=s_{2}=1.4, s_{4}=s_{6}=1.2$ and $s_{7}=s_{8}=1 /(3 v+$ $0.5)$ [24], where $v$ is the kinematic viscosity of the simulated fluid.

$m$ and $m^{e q}$ are vectors of moments. For the D2Q9 scheme, the arrangement of the nine components of the vector $m$ is given in this order [25]:

$$
m=\left(\rho, e, \varepsilon, j_{x}, q_{x}, j_{y}, q_{y}, p_{x x}, p_{x y}\right)^{T}
$$

where $\rho$ is the fluid density, $e$ and $\varepsilon$ are the moments of the energy and the energy square, $q_{x}$ and $q_{y}$ are linked to components of the energy flux and $j=\left(j_{x}, j_{y}\right)=\rho(u, v)$ is the impulsion. $p_{x x}$ and $p_{x y}$ are respectively related to the diagonal and off-diagonal components of the stress tensor. The corresponding equilibrium moments $m_{i}^{e q}$ are [24]:

$$
\begin{aligned}
& m_{0}^{e q}=\rho \\
& m_{1}^{e q}=-2 \rho+3\left(j_{x}^{2}+j_{y}^{2}\right) \\
& m_{2}^{e q}=\rho-3\left(j_{x}^{2}+j_{y}^{2}\right) \\
& m_{3}^{e q}=j_{x} \\
& m_{4}^{e q}=-j_{x} \\
& m_{5}^{e q}=j_{y} \\
& m_{6}^{e q}=-j_{y} \\
& m_{7}^{e q}=\left(j_{x}^{2}-j_{y}^{2}\right) \\
& m_{8}^{e q}=j_{x} j_{y}
\end{aligned}
$$

In contrast to CFD methods, the LB approach does not directly solve the Navier-Stokes equations or the energy equation; it is a statistical method that gives the macroscopic quantities such as the velocity, density, temperature and the pressure as moments, i.e. averages of the microscopic quantities described by the distribution function. For example, the macroscopic fluid variables $\rho$ and $j=$ $\left(j_{x}, j_{y}\right)$ can be directly calculed from the distributions functions given by the D2D9 model as follows:

$$
\rho=\sum_{i=0}^{8} f_{i}, \quad j_{x}=\rho u=\sum_{i=0}^{8} f_{i} c_{i x} \text { and } j_{y}=\rho v=\sum_{i=0}^{8} f_{i} c_{i y}
$$

For the numerical simulation of the acoustic waves propagation, the sound wave is generated by a harmonic function of the fluid density oscillating around the equilibrium density by the following linear relationship [14,26]: 


$$
\rho=\rho_{0}+\rho_{a} \sin (2 \pi f t)
$$

where $\rho_{0}, t, f$ and $\rho_{a}$ are respectively the equilibrium density $\left(\rho_{0}=1\right)$, the time, the frequency and the point source amplitude.

\subsection{D2Q5-MRT for the thermal problem}

For the simulation of natural convection problem, an external force is added in the discrete Boltzmann equation (Eq. (1)) [27]:

$$
F_{i}=w_{i} c_{i} \rho g \beta\left(T-T_{r e f}\right) / c_{s}^{2}
$$

where $w_{i}$ are the weighting factors, $c_{i}$ are the velocities, $g$ is the gravitational vector $\left(\vec{g}=g \overrightarrow{e_{y}}\right), \rho$ is the fluid density, $T$ is the temperature and $T_{\text {ref }}$ is the average temperature $T_{r e f}=\left(T_{\text {hot }}+T_{\text {cold }}\right) / 2$. $\beta$ is the thermal expansion coefficient and $c_{S}$ is the speed of sound $\left(c_{S}=1 / \sqrt{3}\right)$.

As previously mentioned, the temperature field is solved by the D2Q5-MRT. In each node of the LBM lattice, there are five discrete velocities $c_{j}$ (Eq. (12)), as shown in Fig. 2.

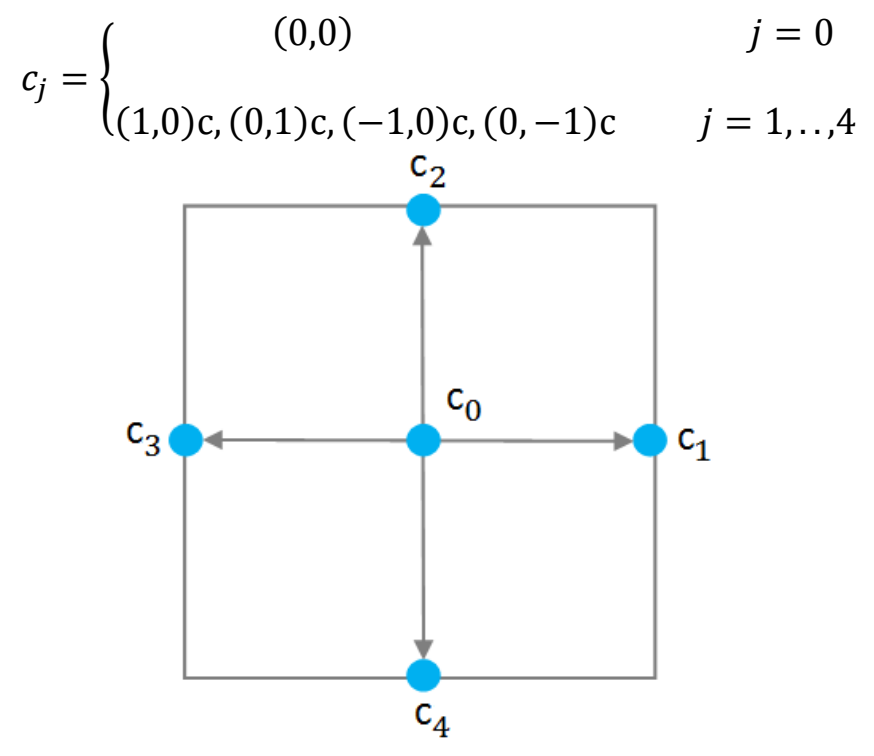

Fig. 2. D2Q5 lattice.

By the same way, we define other distribution functions, noted $g_{j}$, to model the temperature $\mathrm{T}$ as follow [28]:

$$
g_{j}\left(x_{j}+c_{j} \Delta t, t+\Delta t\right)-g_{j}\left(x_{j}, t\right)=-N^{-1} Q\left[n_{j}-n_{j}^{e q}\right]
$$

The transformation matrix $N$ is given by:

$$
N=\left(\begin{array}{rrrrr}
1 & 1 & 1 & 1 & 1 \\
0 & 1 & 0 & -1 & 0 \\
0 & 0 & 1 & 0 & -1 \\
-4 & 1 & 1 & 1 & 1 \\
0 & 1 & -1 & 1 & -1
\end{array}\right)
$$

$Q$ is the diagonal relaxation matrix of the thermal problem, and it can be written as: 


$$
\mathrm{Q}=\operatorname{diag}\left(q_{0}, q_{1}, q_{2}, q_{3}, q_{4}\right)
$$

In the present simulation, we choose $q_{0}=1, q_{1}=q_{2}=6 /(3+\sqrt{3})$ and $q_{3}=q_{4}=1.5$ [29].

For the D2Q9 model, there are three conserved quantities: $\rho, j_{x}$ and $j_{y}$. However, for the D2Q5 model, the temperature $T$ is the only conserved quantity and is obtained by:

$$
T=\sum_{j=0}^{4} g_{j}
$$

The equilibrium moments, $n_{j}^{e q}$ corresponding to the five distribution functions $g_{j}$, are discussed in details in [30]:

$$
n_{0}^{e q}=T, n_{1}^{e q}=u T, n_{2}^{e q}=v T, n_{3}^{e q}=a T \text { and } n_{4}^{e q}=0
$$

where the constant $a$ is related to the thermal diffusivity $\alpha$ by the following equation:

$$
\alpha=\frac{(4+\mathrm{a})}{10}\left(\frac{1}{q_{1}}-\frac{1}{2}\right)
$$

It should be noted that the factor $a$ must be less than one to avoid instability of the D2Q5-LBM scheme [31].

\section{Boundary conditions}

The thermal boundary conditions employed in this work are discussed in details in [25]. For the vertical isothermal walls, the anti-bounce-back boundary conditions are applied: $g_{j}=g_{j}^{-}-2 T_{w}(1+$ $a / 4) / 5$, where $T_{w}$ represents the cold and hot temperatures and $g_{j}^{-}$is the distribution function in the opposite direction of $g_{j}$. The adiabatic conditions are used on the horizontal walls of the cavity $: g_{2}=g_{4}$.

For the distribution functions $f_{i}$, there are different types of boundary conditions in the literature to define the solid walls. The best known are bounce-back conditions [14,27]. The unknown distribution functions are determined from the known functions. For example, at the east and west boundaries of the cavity (see Fig. 3), the components $f_{3}, f_{6}, f_{7} f_{1}, f_{5}$ and $f_{8}$ are calculated as follows: at the east boundary, $f_{3}=f_{1}, f_{6}=f_{8}, f_{7}=f_{5}$ and at the west boundary, $f_{1}=f_{3}, f_{5}=f_{7}$ and $f_{8}=f_{6}$.

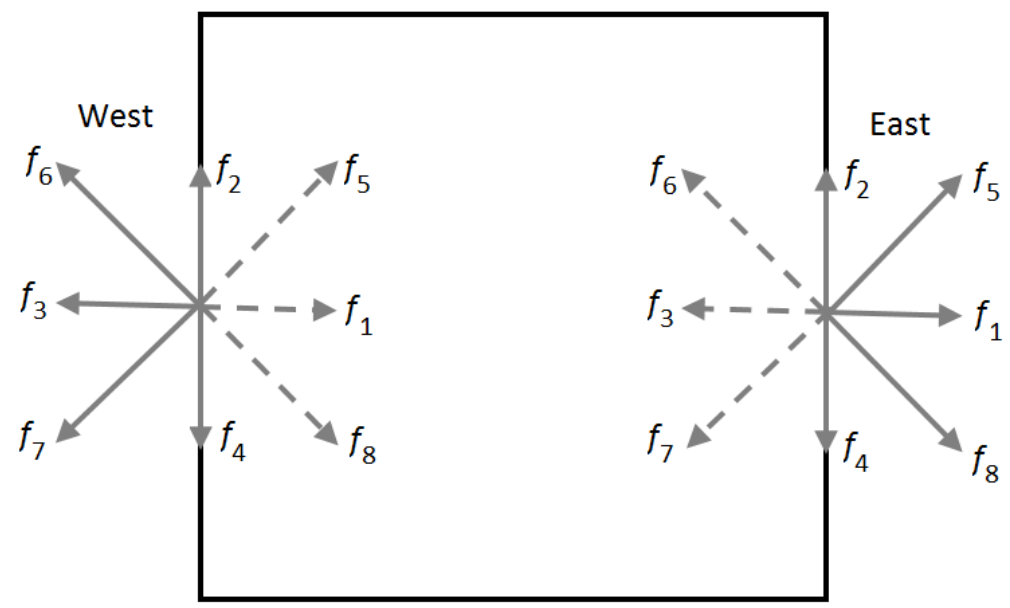

Fig. 3. Boundary conditions used. 


\section{Results and Discussion}

We started by validating our code. For that, we tested the performances of our numerical model by comparing the results that we found with those reported in the literature [14,25]:

\section{First test: propagation of the sound wave in an unheated square cavity}

First, calculations were performed for the problem studied by Salomons and Lohman [14], who have modeled the propagation of sound waves inside a square enclosure. These waves are generated by a point source, located at the center of this enclosure (Fig. 4).

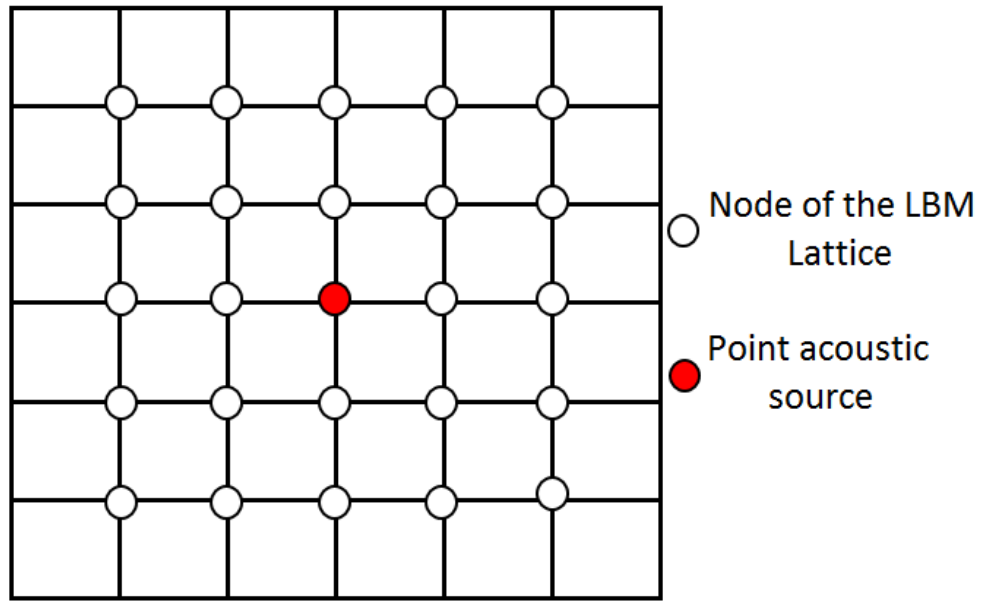

Fig. 4. Point acoustic source located at the center of the LBM lattice.

The grid size used is $2000 \times 2000$ and the point source is located at the node $(1000,1000)$. The physical parameters used here, in LBM units, are: $v=0.06, t=1600, \rho_{a}=0.01$ and $\mathrm{T}=40$. The obtained results are shown in Fig. 5. Let us note an excellent agreement between these results.
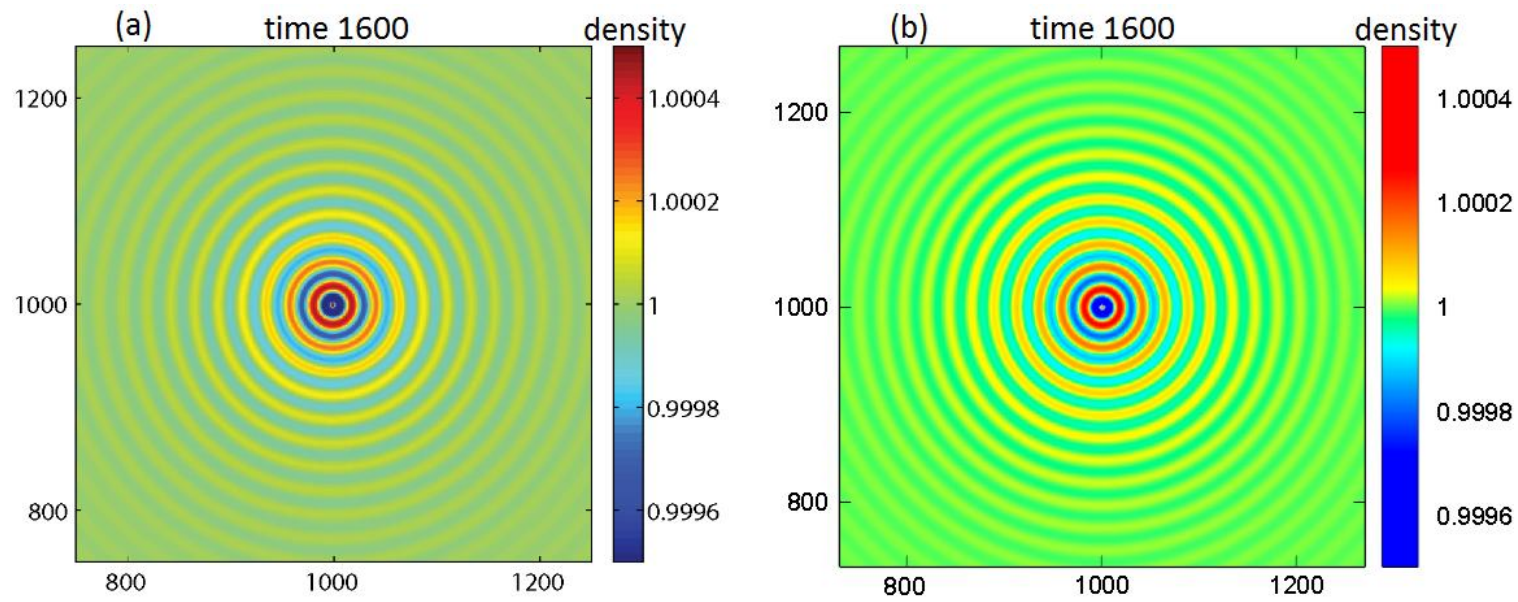

Fig. 5. Sound wave propagating in a square cavity at time 1600: (a) reference results [14]; (b) our results.

We can also validate our results through the analytic solution of the density. This analytic solution is given by the real part of the following equation $[14,26]$ :

$$
\rho(r, t)=1+A H_{0}^{(2)}(k r) e^{j \omega t}
$$


where $A$ is a constant and $H_{0}^{(2)}$ is the Hankel function of order zero and second kind. $H_{0}^{(2)}$ depends on wave number $k$ and distance $r$ to the acoustic source, it can be expressed as follows [26]:

$$
H_{0}^{(2)}(k r)=J_{0}(k r)-j Y_{0}(k r)
$$

where $J_{0}$ and $Y_{0}$ are Bessel functions of the first and second kinds, respectively.

The factor $\mathrm{A}$ is a constant which depends on the amplitude of the point acoustic source. Its mathematical expression is discussed in detail in the reference [26].

Salomons and Lohman [14] showed that the wave dissipates with distance due to the fluid viscosity. This dissipation is represented by a coefficient noted $\alpha$ :

$$
\alpha=\frac{1}{2} \omega^{2}\left(\frac{4}{3} v+v_{B}\right) c_{S}^{-3}
$$

where $v_{B}$ is the Bulk viscosity $\left(v_{B}=\frac{2}{3} v\right)$.

To take into account the dissipation in the analytical solution, the attenuation coefficient $\alpha$ is integrated into the wave number $k$ [14]:

$$
k=\frac{2 \pi}{\lambda}-j \alpha
$$

where $\lambda$ is the wavelength.

The results obtained from the analytical solution are illustrated in Fig. 6. These results were calculated using equation (19) containing the Hankel function which is not defined at the source position $(r=0)$. Consequently, this produces a deviation between the analytical and numerical solutions at this point. According to the reference [26], this is considered a limitation of the point acoustic source method.

There also, an excellent agreement is noted between the density fields in Figs. 5 and 6(a) and between the density profiles along the x-axis in Fig. 6(b) which presents the exact and the LB solutions in the same graph. From this figure, the absolute error can be measured to show the error between the analytical and numerical calculations [32]. It can be calculated as the difference between the analytical $\left(\rho_{\text {Anl }}\right)$ and numerical $\left(\rho_{\text {Num }}\right)$ densities:

$$
E a=\left|\rho_{A n l}-\rho_{N u m}\right|
$$
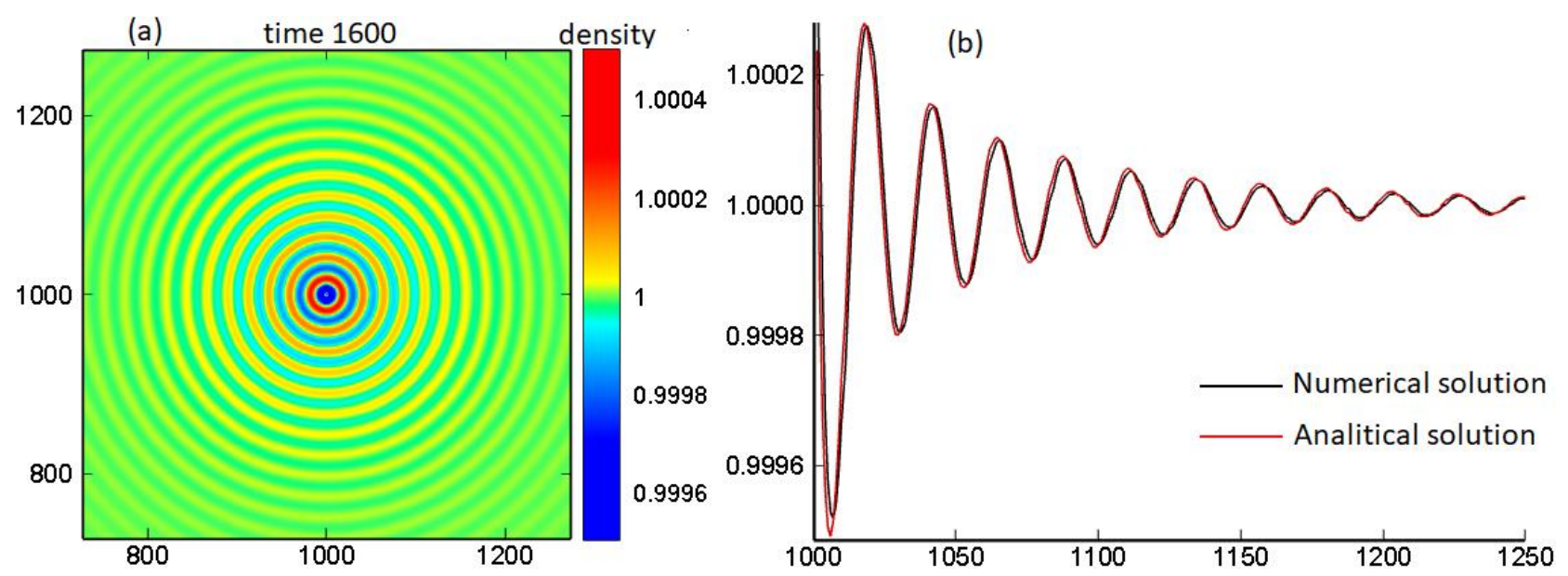

Fig. 6. Analytic solution at time 1600: (a) density field; (b) cross-section at $y=1001$ of the density profiles of the analytic and numerical solutions along the $\mathrm{x}$-axis. 
The variation of the absolute error along the $\mathrm{x}$-axis is depicted in Fig. 7. The result is a waveform that almost oscillates between 0 and $10^{-4}$ and attenuates with distance away from the source. These values indicate that the error is very small, and consequently the numerical results are very close to those obtained with the density analytical solution. Also, the mean absolute error (En) can be calculated as being the mean of the absolute error. Its value found from this calculation is $4.32510^{-6}$.

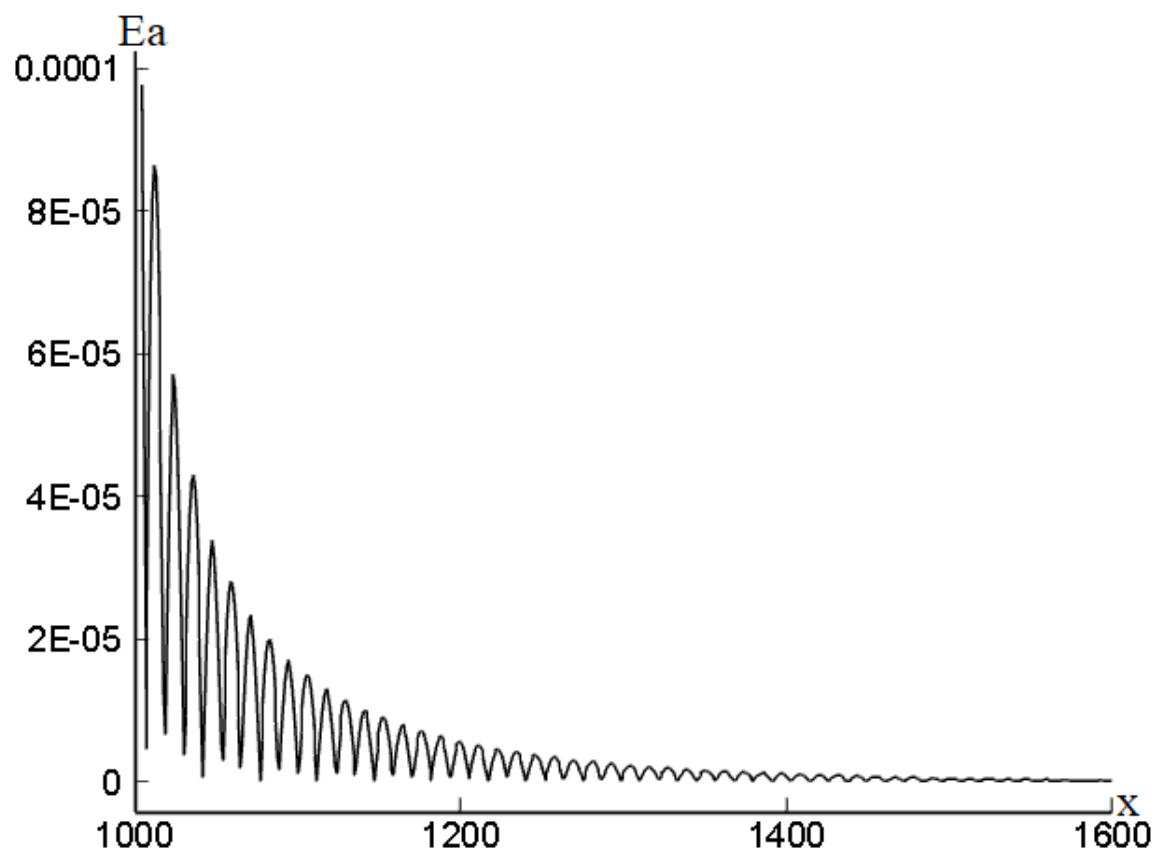

Fig. 7. Variation of the absolute error along the $\mathrm{x}$-axis at time 1600.

\section{Second test: Free convection in a square differentially heated cavity}

For the natural convection phenomena, the code was extensively confronted with benchmark problems to check its validity. The physical problem studied is an empty square enclosure. Its vertical walls are isothermal and held at different temperatures, whereas the top and bottom walls are adiabatic. The investigation is performed for a Rayleigh number ranged between $10^{3}$ and $10^{8}$ and for a Prandtl number fixed at 0.71 .

Table 1 shows comparisons on the average Nusselt number $\left\langle N_{u}\right\rangle$, the maximum vertical and horizontal velocities $v_{\text {max }}$ and $u_{\text {max }}$, respectively, for different Rayleigh numbers. For the temperature and the hydrodynamic fields, we show isotherms and streamlines for $\mathrm{Ra}=10^{3}$ and $\mathrm{Ra}=10^{8}$ in Fig. 8, as examples for the laminar and transitional flow regimes, respectively. The present numerical results show that the laminar and transitional flow regimes are well predicted by the calculations performed. These results are also in good agreement with those published previously. Consequently, we can say that our proposed model is efficient, precise and numerically stable at all Rayleigh numbers. Then, it is used to study the effect of thermal convection on the propagation of sound waves.

The average Nusselt number $\left\langle N_{u}\right\rangle$ is calculated at the hot wall by the following equation:

$$
\left\langle N_{u}\right\rangle=\frac{1}{H} \int_{0}^{H} N_{u} d y
$$

where $H$ is the height of the cavity and $N_{u}$ is the local Nusselt number which is given by the following equation:

$$
N_{u}=-\frac{H}{T_{h}-T_{c}}\left(\frac{\partial T}{\partial x}\right)_{\text {wall }}
$$


Table 1

Results comparison.

\begin{tabular}{ccccccc}
\hline $\mathrm{Ra}$ & \multicolumn{2}{c}{ Kuznik et al. [33] } & Dixit et al. [34] & Mezrhab et al. [25] & Jami et al. [30] & Our results \\
\hline $\mathbf{1 0}^{\mathbf{3}}$ & $\left\langle\boldsymbol{N}_{\boldsymbol{u}}\right\rangle$ & 1.117 & 1.1272 & 1.112 & 1.115 & 1.123 \\
& $\boldsymbol{u}_{\text {max }}$ & 3.636 & 3.6529 & 3.667 & 3.658 & 3.650 \\
& $\boldsymbol{v}_{\text {max }}$ & 3.686 & 3.6820 & 3.714 & 3.712 & 3.697 \\
& & & & & & \\
$\mathbf{1 0}^{\mathbf{4}}$ & $\left\langle\boldsymbol{N}_{\boldsymbol{u}}\right\rangle$ & 2.246 & 2.247 & 2.241 & 2.246 & 2.255 \\
& $\boldsymbol{u}_{\text {max }}$ & 16.167 & 16.163 & 16.202 & 16.149 & 16.191 \\
& $\boldsymbol{v}_{\text {max }}$ & 19.597 & 19.569 & 19.644 & 19.684 & 19.642 \\
& & & & & & \\
$\mathbf{1 0}^{\mathbf{5}}$ & $\left\langle\boldsymbol{N}_{\boldsymbol{u}}\right\rangle$ & 4.518 & 4.5226 & 4.519 & 4.525 & 4.538 \\
& $\boldsymbol{u}_{\text {max }}$ & 34.962 & 35.521 & 34.805 & 34.557 & 34.769 \\
& $\boldsymbol{v}_{\text {max }}$ & 68.578 & 68.655 & 68.630 & 68.624 & 68.755 \\
& & & & & & \\
$\mathbf{1 0}^{\mathbf{6}}$ & $\left\langle\boldsymbol{N}_{\boldsymbol{u}}\right\rangle$ & 8.792 & 8.805 & 8.817 & 8.825 & 8.814 \\
& $\boldsymbol{u}_{\text {max }}$ & 64.133 & 64.186 & 64.793 & 64.549 & 64.319 \\
& $\boldsymbol{v}_{\text {max }}$ & 220.537 & 219.866 & 219.663 & 220.915 & 220.085 \\
\hline
\end{tabular}
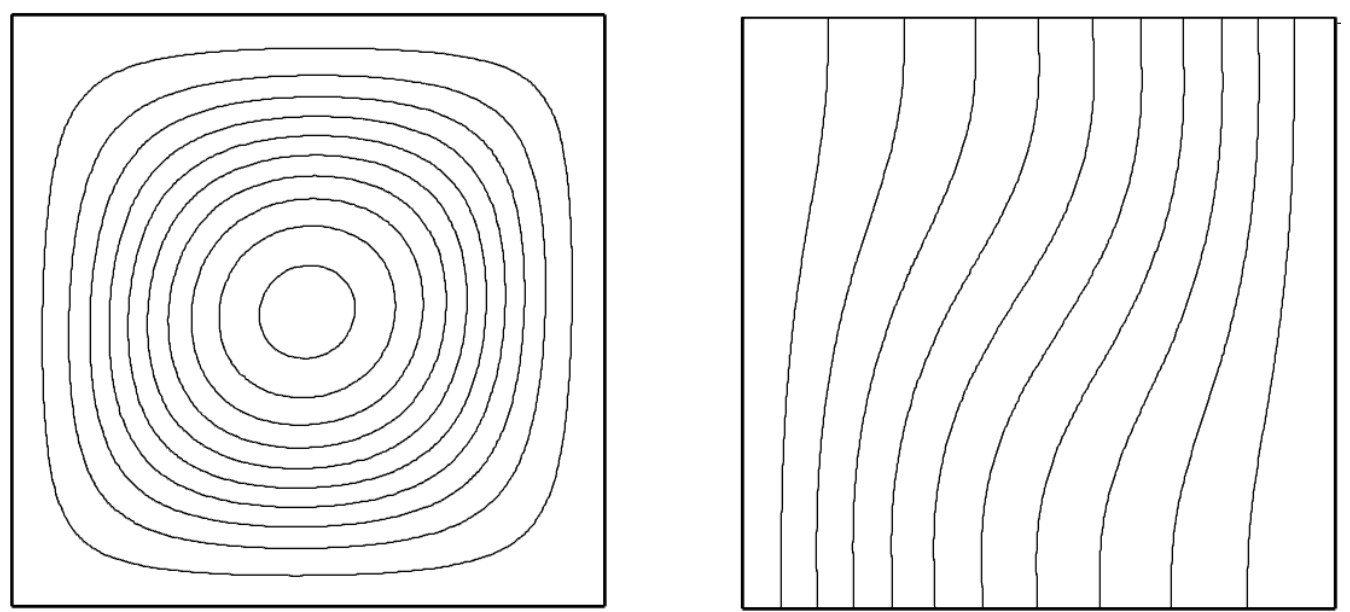

$\mathrm{Ra}=10^{3}$
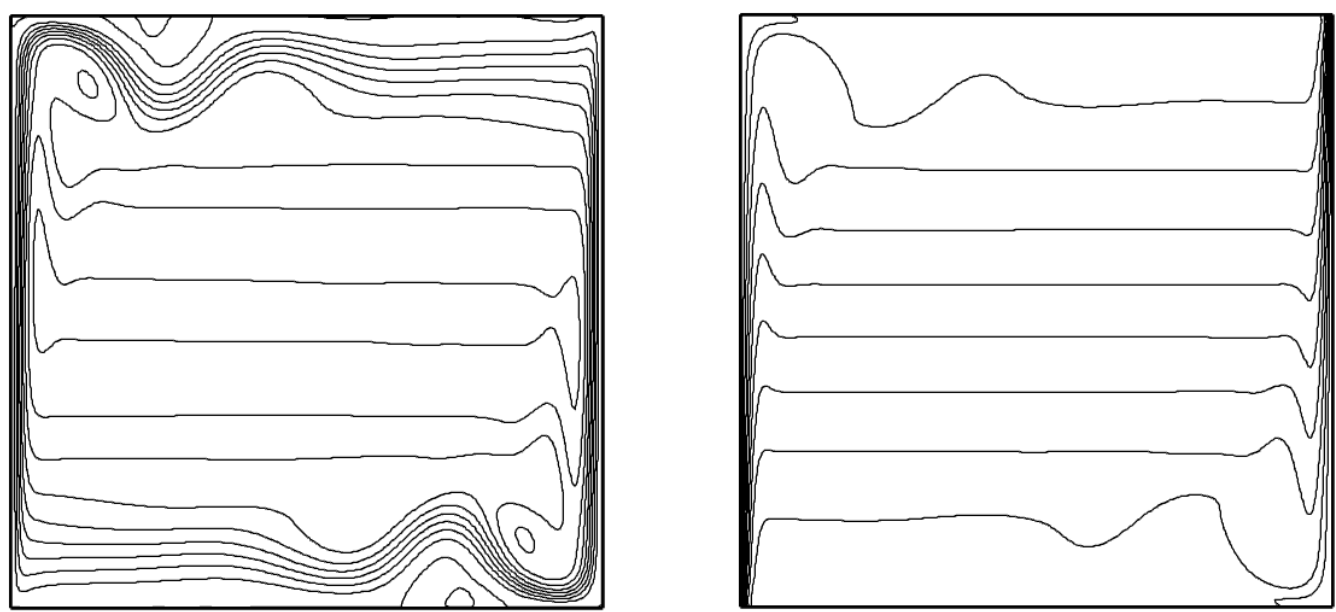

$\mathrm{Ra}=10^{8}$

Fig. 8. Streamlines (left) and isotherms (right) for Rayleigh numbers $\mathrm{Ra}=10^{3}$ and $\mathrm{Ra}=10^{8}$. 


\section{Propagation of the sound wave in a differentially heated square enclosure}

The main purpose of the present work is to study the effect of the natural convection on the propagation of acoustic waves generated by a point acoustic source located at the center of a square differentially heated enclosure filled with air (Fig. 9). The top and bottom walls are adiabatic and the vertical isothermal walls are maintained at two dimensionless temperatures $T_{h}=0.5$ and $T_{c}=-0.5$.

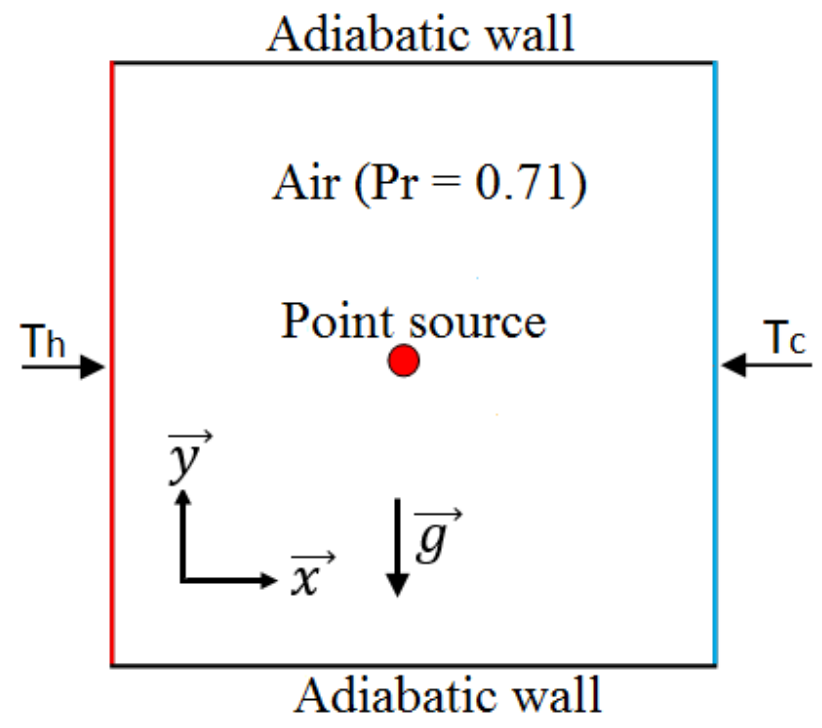

Fig. 9. Geometry of the studied physical problem.

The acoustic waves and the heat transfer are computed using respectively the D2Q9 and D2Q5 lattice Boltzmann models. We remember that in contrast to CFD methods, the LB approach does not directly solve the Navier- Stokes equations or the energy equation. In fact, it is a statistical method that gives the macroscopic quantities such as the velocity, density, temperature, and the pressure as moments, that is, averages of the microscopic quantities described by the distribution function (see Equation 9). These quantities are used to assess the effects of natural convection on the wave propagation. Then, the main equations governing the problem are Equations (1) and (13).

Figure 10 shows the density distributions for different Rayleigh numbers and for a LBM viscosity fixed at 0.02 . Let us note that when the heat is exchanged mainly by conduction between the isothermal walls $\left(\mathrm{Ra}=10^{3}\right)$, the density keeps the shape obtained without heat exchange $(\mathrm{R} a=0)$. Indeed, it presents circular bands whose radius increases while moving away from the source. Therefore, we can say that for this thermal regime, there is no effect of natural convection on the propagation of sound waves. For $\mathrm{Ra}=10^{4}$, the convection is still low compared to conduction and the thermal effect on the wave is always weak. The effect of the temperature on the propagation of sound wave begins to appear in the extremities of the cavity when the convection became important $\left(\mathrm{Ra}=10^{5}\right)$. When Ra reaches $10^{6}$, the wave propagation begins to be limited in a central region at mid height of the cavity without arriving at the horizontal walls. For a Rayleigh number equal to $10^{7}$, the density tends to become horizontally organized and a vertical gradient of density appears in the boundary layers near the horizontal walls. In addition, for the LBM period and viscosity used in this calculation, we can say that for $\mathrm{Ra}=10^{6}$ and $10^{7}$, the pressure gradients created by thermal convection (Fig. 11) prevent the propagation of the wave towards the horizontal walls.

The density variation can be also depicted by profiles along the central $\mathrm{x}$ and $\mathrm{y}$-axes (Fig. 12). Along the $\mathrm{x}$-axis, the density has the shape of a wavy curve and has only one maximum at the acoustic source; in contrast, two other maxima are observed at the top and bottom walls along the y-axis. 
It should be noted that in all calculations, the time 600000 was used to stabilize the thermal convection and the interference phenomenon exists because the acoustic source used in this study depends on time (Eq. (10)) that is represented by the number of iterations in the LBM code. Thus, if the number of iterations is greater than the number of grid nodes in one direction, the interference effect between the direct sound waves and the waves reflected by the walls of the cavity will occur (see an example in Fig. 13).
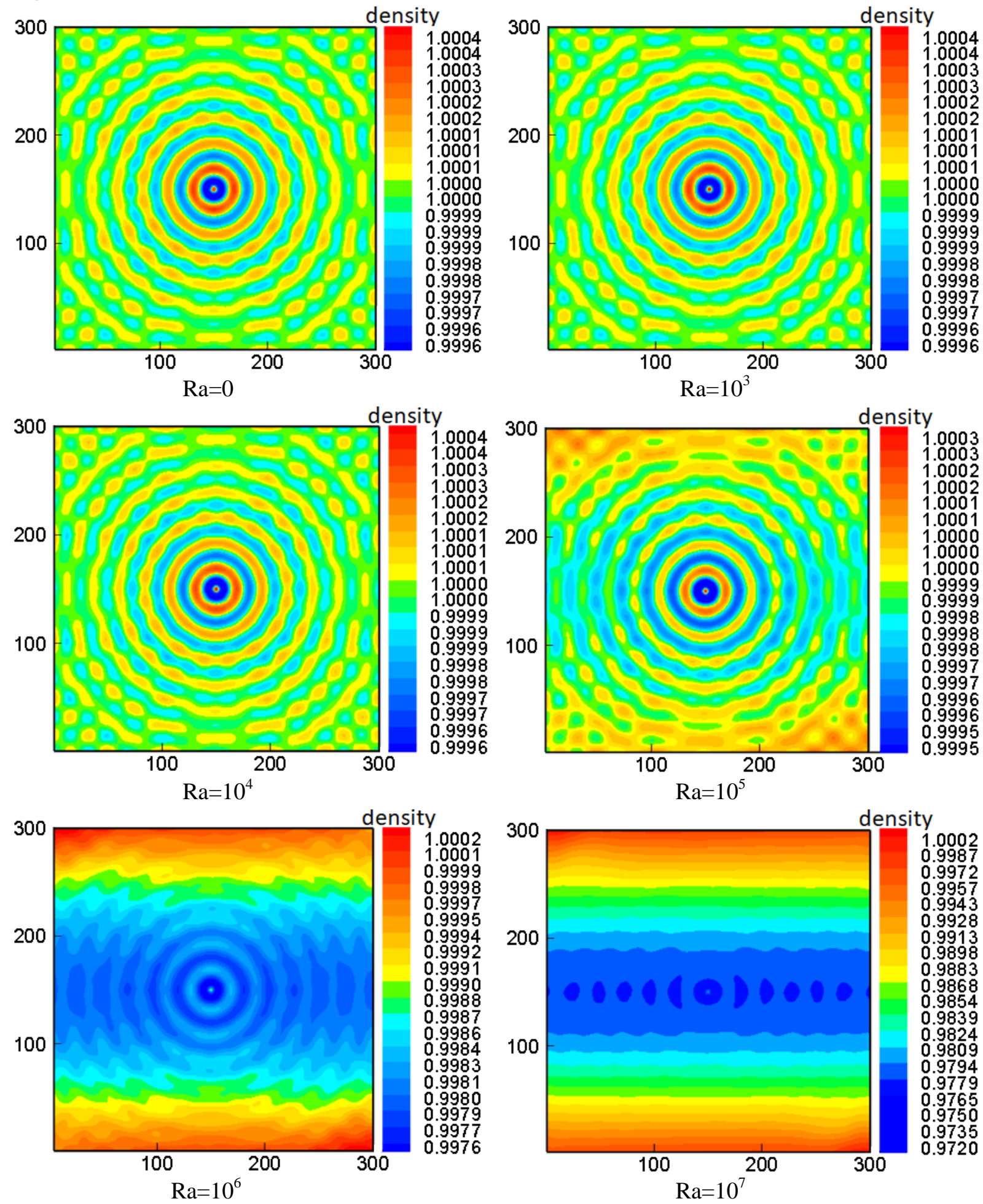

Fig. 10. Propagation of sound wave in a square differentially heated cavity at time 500000 for Rayleigh number ranging from $10^{3}$ to $10^{8}$ with $\rho_{a}=0.01$ and $\mathrm{T}=40$. 

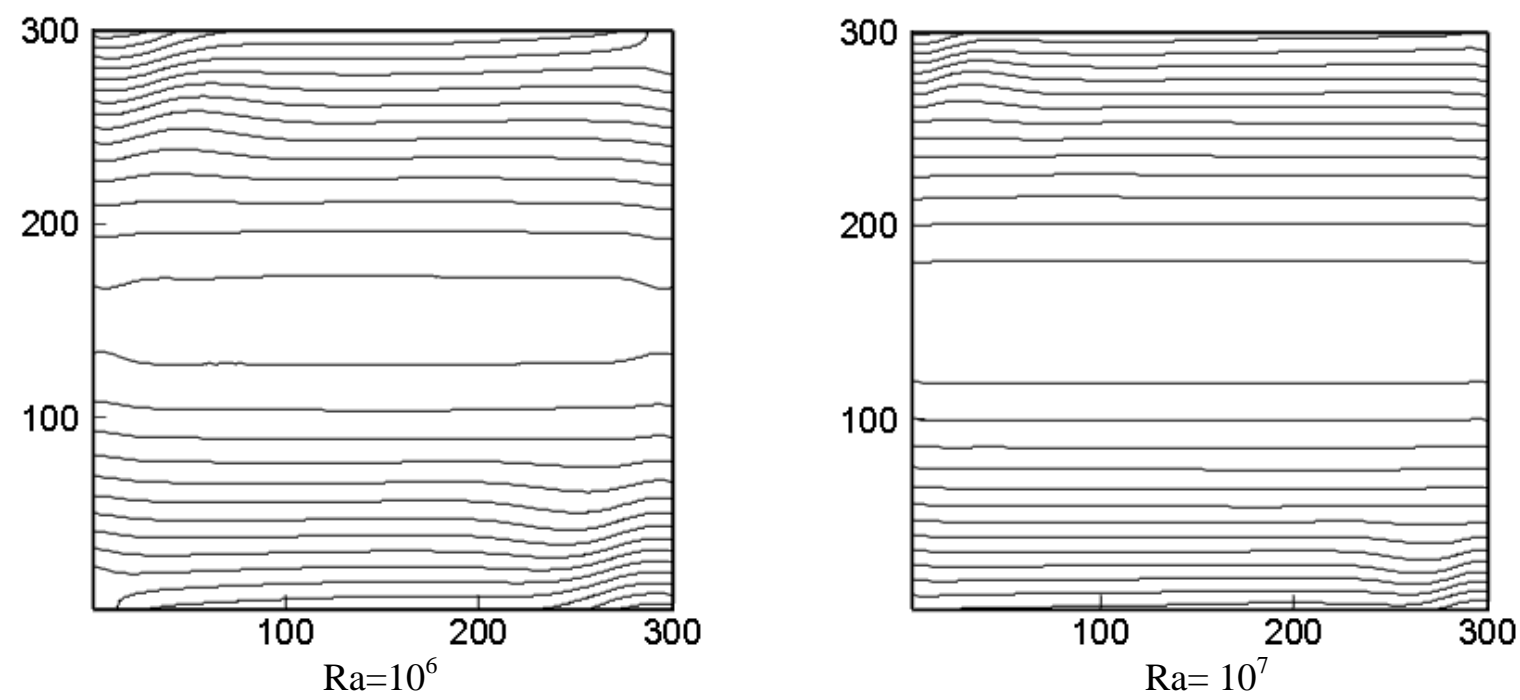

Fig. 11. Contours of the pressure due to the natural convection without acoustic waves for $R a=10^{5}$ and
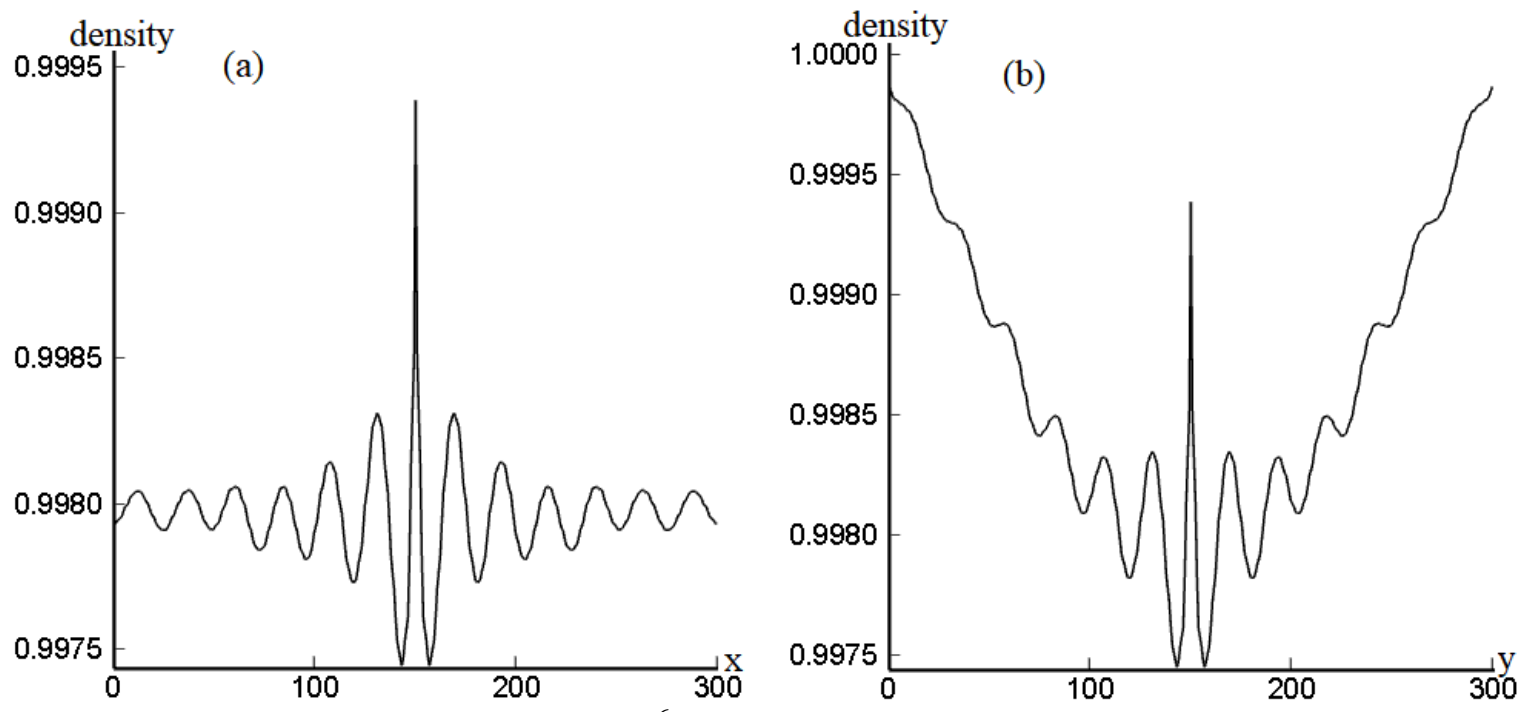

Fig. 12. Density profiles for $\mathrm{Ra}=10^{6}$ along the $\mathrm{x}(\mathrm{a})$ and $\mathrm{y}(\mathrm{b})$ axes at the time 600000 .
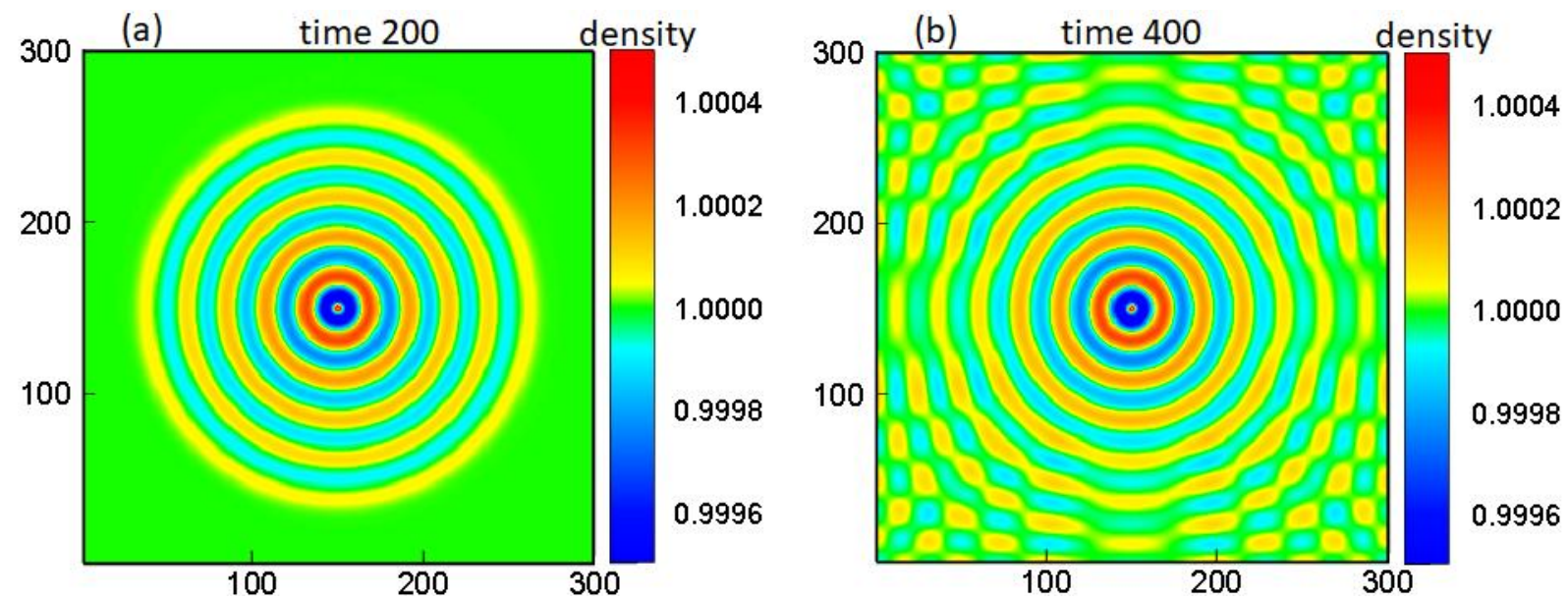

Fig. 13. Propagation of a sound wave in an unheated square cavity without (a) and with interference (b) at times 200 and 400 , respectively. 
Figure 14 presents a calculation of the coupling of the velocity field produced by the point source with the one created with natural convection for an amplitude equal to 0.01. For a low value of Rayleigh number $\left(\mathrm{Ra}=10^{3}\right)$, the field created by the wave is driven by that created by thermal convection. When the Rayleigh number becomes large $\left(\mathrm{Ra}=10^{5}\right.$ ), we can see that the velocity field produced by the sound source is invisible because the convective contribution is important compared to the contribution of the acoustic wave to the velocity field. The acoustic contribution would be clearly visible if the wave amplitude is important.

Figure 15 shows a test case with an amplitude of 0.08 . The obtained results show that there is no convective contribution to the velocity field for $\mathrm{Ra}=10^{3}$. The configuration found for this Rayleigh number retains the shape of that found without convection $(\mathrm{Ra}=0)$. The natural convection training of the flow created by the point source is dominated when the Rayleigh number becomes important $(\mathrm{Ra}=$ $10^{5}$ ).

It should be noted that, for the acoustic model used in our simulation, the sound wave amplitude must be very low compared to the equilibrium density in order to avoid the effects of non-linear waves [26]. 0.08 is always low compared to the LBM equilibrium density, which is equal to one.

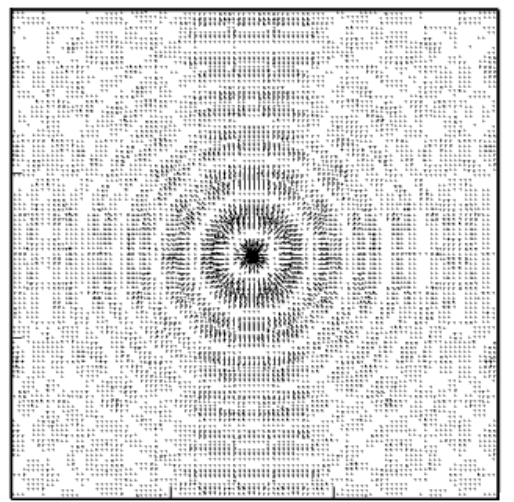

$\mathrm{Ra}=0$

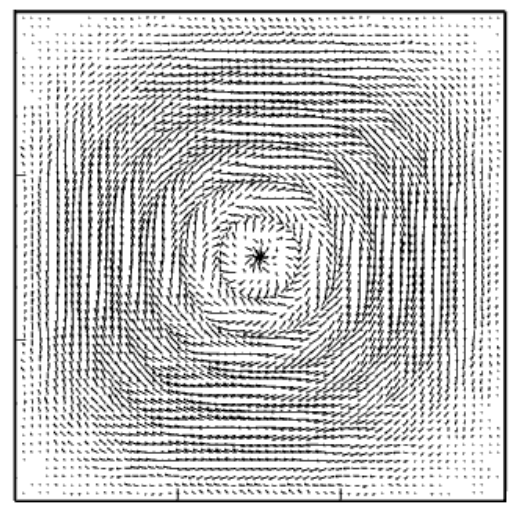

$\mathrm{Ra}=10^{3}$

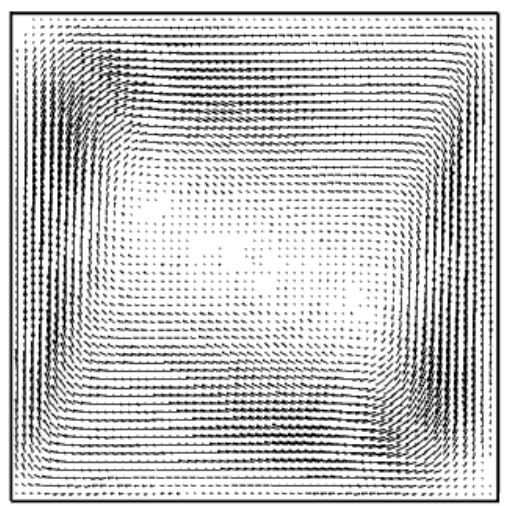

$\mathrm{Ra}=10^{5}$

Fig. 14. Velocity fields found with a LBM amplitude of 0.01 .

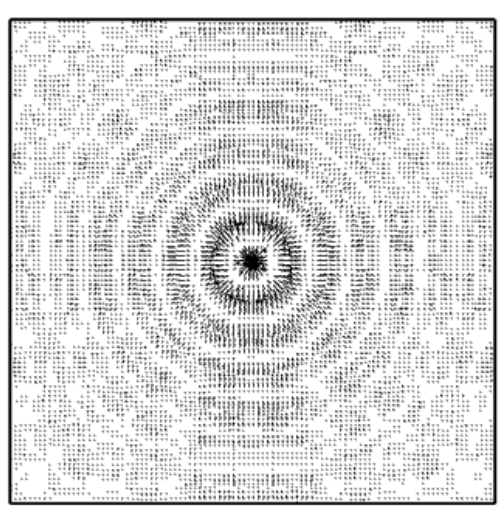

$\mathrm{Ra}=0$

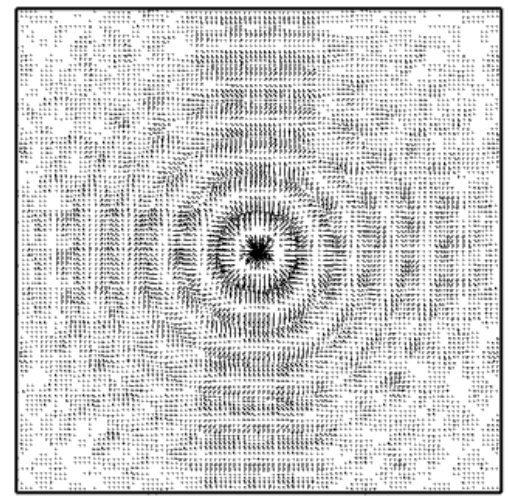

$\mathrm{Ra}=10^{3}$

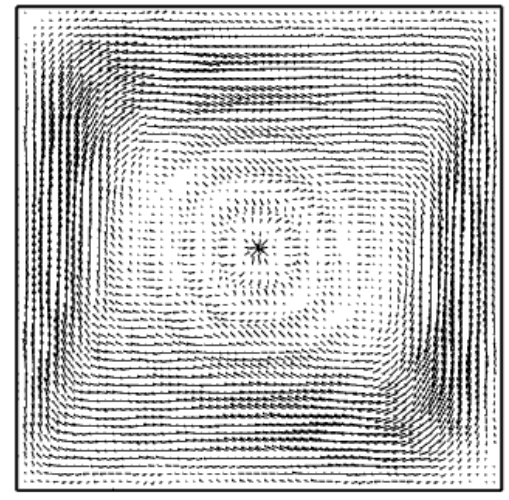

$\mathrm{Ra}=10^{5}$

Fig. 15. Velocity fields obtained with an amplitude of 0.08 . 


\section{Conclusions}

The results presented in this work are directly related to the fluid density. The variation of this density is caused by two different phenomena: the heat transfer and the propagation of sound waves. Concerning the heat transfer, the variation of density is due to the variation of temperature and for acoustic waves, the density variation depends on the amplitude and frequency of the wave. For the conditions considered in this study, we can conclude that, for an amplitude value equal to 0.01 , the effect of natural convection on the propagation of sound waves is negligible for $\mathrm{Ra} \leq 10^{4}$. The effect begins to appear for $\mathrm{Ra}=10^{5}$ and becomes more pronounced for $\mathrm{Ra} \geq 10^{6}$. For the amplitude value equal to 0.08 , this effect becomes visible for $\mathrm{Ra} \geq 10^{6}$. Then, the interaction between the natural convection and the wave propagation depends mainly on the Rayleigh number and the amplitude of the wave.

The study represented in this work will be the beginning of another LBM study of the coupling of thermal convection with acoustic waves, in particular the combination of the flow generated by acoustic waves (acoustic streaming) with that produced by convection [35,36]. The resulting flux created can be used to purify photovoltaic silicon [37]. The point source will be replaced by a rectangular acoustic source to generate an acoustic beam then the force induced by the sound source (acoustic force) will be used to digitally produce the acoustic streaming. Also, the proposed numerical model can be used in the case of ultrasonic waves and this can be the subject of future studies. 


\section{References}

[1] X. He and L. S. Luo, Theory of the lattice Boltzmann method: From the Boltzmann equation to the lattice Boltzmann equation. Physical Review E, 1997, vol. 56, no 6, p. 6811.

[2] U. Frisch, B. Hasslacher, Y. Pomeau. Lattice-gas automata for the Navier-Stokes equation. Physical review letters, 1986, vol. 56, no 14, p. 1505.

[3] G. R. McNamara, G. Zanetti. Use of the Boltzmann equation to simulate lattice-gas automata. Physical review letters, 1988, vol. 61, no 20, p. 2332.

[4] L. Huang. A lattice Boltzmann approach to acoustic-wave propagation. Advances in Geophysics, 2007, vol. 48, p. 517-559.

[5] Y. Guangwu, C. Yaosong, H. Shouxin. Simple lattice Boltzmann model for simulating flows with shock wave. Physical review E, 1999, vol. 59, no 1, p. 454.

[6] S. Xiao. A lattice Boltzmann method for shock wave propagation in solids. Communications in Numerical Methods in Engineering, 2007, vol. 23, no 1, p. 71-84.

[7] N. G. Frantziskonis. Lattice Boltzmann method for multimode wave propagation in viscoelastic media and in elastic solids. Physical Review E, 2011, vol. 83, no 6, p. 066703.

[8] G. S. O’Brien, S. Gareth, T. Nissen-Meyer, C.J. Bean. A lattice Boltzmann method for elastic wave propagation in a poisson solid. Bulletin of the Seismological Society of America, 2012, vol. 102, no 3, p. 1224-1234.

[9] X. M. Li, X, R. C. K. Leung, R. M. C. So. One-step aeroacoustics simulation using lattice Boltzmann method. AIAA journal, 2006, vol. 44, no 1, p. 78-89.

[10] S. Marié, D. Ricot, P. Sagaut. Comparison between lattice Boltzmann method and Navier-Stokes high order schemes for computational aeroacoustics. Journal of Computational Physics, 2009, vol. 228, no 4, p. 1056-1070.

[11] D. Haydock, J. M. Yeomans. Lattice Boltzmann simulations of acoustic streaming. Journal of Physics A: Mathematical and General, 2001, vol. 34, no 25, p. 5201.

[12]D. Haydock, J. M. Yeomans. Lattice Boltzmann simulations of attenuation-driven acoustic streaming. Journal of Physics A: Mathematical and General, 2003, vol. 36, no 20, p. 5683.

[13] J. M. Buick, C. A. Greated, D. M. Campbell. Lattice BGK simulation of sound waves. EPL (Europhysics Letters), 1998, vol. 43, no 3, p. 235.

[14] E. M. Prandtl mons, W. J. A. Lohman, Simulation of sound waves using the lattice Boltzmann method for fluid flow: Benchmark cases for outdoor sound propagation, PloS one, 2016, vol. 11, no 1, p. e0147206.

[15] M. Xia, S. Wang, H. Zhou, X. Shan, H. Chen, Q. Li, Q. Zhang. Modelling viscoacoustic wave propagation with the lattice Boltzmann method. Scientific Reports, 2017, vol. 7, no 1, p. 10169.

[16] W. G. Vincenti, B. S. Baldwin. Effect of thermal radiation on the propagation of plane acoustic waves. Journal of Fluid Mechanics, 1962, vol. 12, no 3, p. 449-477.

[17] J. H. Park, S. W. Baek. Effects of thermal radiation on the sound wave propagation in a gasparticle two-phase medium. Numerical Heat Transfer: Part A: Applications, 2000, vol. 38, no 4, p. 341-355.

[18] C. Tarau, V. Sheverev, G. Vradis. Temperature gradient effects on sound wave propagation. In : 42nd AIAA Aerospace Sciences Meeting and Exhibit. 2004. p. 366.

[19] M. Nabavi, K. Siddiqui, J. Dargahi. Effects of transverse temperature gradient on acoustic and streaming velocity fields in a resonant cavity. Applied Physics Letters, 2008, vol. 93, no 5, p. 051902.

[20] E. Saint Ellier, Y. Bailly, L. Girardot, D. Ramel, P. Nika. Temperature gradient effects on acoustic and streaming velocities in standing acoustic waves resonator. Experimental Thermal and Fluid Science, 2015, vol. 66, p. 1-6. 
[21] M. Červenka, M. Bednařík. Numerical study of the influence of the convective heat transport on acoustic streaming in a standing wave. The Journal of the Acoustical Society of America, 2018, vol. 143, no 2, p. 727-734.

[22] M. E. Viggen, The lattice Boltzmann method: Fundamentals and acoustics, thesis, Norwegian University of Science and Technology, 2014.

[23] X. Guo, C. Zhong, C. Zhuo, J. Cao. Multiple-relaxation-time lattice Boltzmann method for study of two-lid-driven cavity flow solution multiplicity. Theoretical and Computational Fluid Dynamics, 2014, vol. 28, no 2, p. 215-231.

[24] A. A. Mohammed, Lattice Boltzmann method: fundamentals and engineering applications with computer codes, Springer Science \& Business Media, 2011.

[25] A. Mezrhab, M. Amine Moussaoui, M. Jami, H. Naji, M. H. Bouzidi, Double MRT thermal lattice Boltzmann method for simulating convective flows, Physics Letters A, 2010, vol. 374, no 34, p. 3499-3507.

[26] E. M. Viggen. The lattice Boltzmann method with applications in acoustics. 2009. Thèse de maîtrise. Norges teknisk-naturvitenskapelige universitet, Fakultet for naturvitenskap og teknologi, Institutt for fysikk.

[27] A. A. Mohamad, A. Kuzmin. A critical evaluation of force term in lattice Boltzmann method, natural convection problem. International Journal of Heat and Mass Transfer, 2010, vol. 53, no 5-6, p. 990-996.

[28] J. Wang, D. Wang, P. Lallemand, L. S. Luo. Lattice Boltzmann simulations of thermal convective flows in two dimensions. Computers \& Mathematics with Applications, 2013, vol. 65, no 2, p. 262-286.

[29] B. Trouette. Lattice Boltzmann simulations of a time-dependent natural convection problem. Computers \& Mathematics with Applications, 2013, vol. 66, no 8, p. 1360-1371.

[30] M .Jami, F. Moufekkir, A. Mezrhab, J. P. Fontaine, M. H. Bouzidi. New thermal MRT lattice Boltzmann method for simulations of convective flows. International Journal of Thermal Sciences, 2016, vol. 100, p. 98-107.

[31] Z. Li, M. Yang, Y. Zhang. Double MRT thermal lattice Boltzmann method for simulating natural convection of low Prandtl number fluids. International Journal of Numerical Methods for Heat \& Fluid Flow, 2016, vol. 26, no 6, p. 1889-1909.

[32] F. John, G. Mathematician. Lectures on advanced numerical analysis. New York : Gordon and Breach, 1967.

[33] F. Kuznik, J. Vareilles, G. Rusaouen, G. Krauss. A double-population lattice Boltzmann method with non-uniform mesh for the simulation of natural convection in a square cavity. International Journal of Heat and Fluid Flow, 2007, vol. 28, no 5, p. 862-870.

[34] H. N. Dixit, V. Babu. Simulation of high Rayleigh number natural convection in a square cavity using the lattice Boltzmann method. International journal of heat and mass transfer, 2006, vol. 49, no 3-4, p. 727-739.

[35] M. C. Charrier-Mojtabi, X. Jacob, T. Dochy, A. Mojtabi. Species separation of a binary mixture under acoustic streaming. The European Physical Journal E, 2019, vol. 42, no 5, p. 64.

[36] H. Lei, D. Henry, H. BenHadid. Numerical study of the influence of a longitudinal sound field on natural convection in a cavity. International journal of heat and mass transfer, 2006, vol. 49, no 19-20, p. 3601-3616.

[37] B. Moudjed. Caractérisation expérimentale et théorique des écoulements entrainés par ultrasons. Perspectives d'utilisation dans les procédés de solidification du silicium photovoltaïque. 2013. Doctoral thesis. INSA, Lyon. 
